\title{
Numerical simulation of an RF asymmetric nonthermal plasma reactor used for plasma polymerization
}

\author{
S Elaissi*, H Alyousef \\ College of Sciences - Princess Nourah Bint Abdulrahman \\ University - Riyadh - KSA
}

\begin{abstract}
The aims of this paper is to investigate the control of plasma properties via the geometrical asymmetry effect in a capacitive coupled discharge used for polymer processing. The simulation results prove that the bulk position and density profiles of positive ions, negative ions, and electrons have a clear dependence on geometric asymmetry effect. The underlying mechanisms identified shows a more collisional sheath at the smaller powered surface due to the larger sheath width, and a higher energy at the smaller surface due to the higher mean sheath voltage compared to the larger surface. The argon modelling results are compared to experimental results from the literature for a range of operating conditions. The results show that the argon model results can be used to predict the plasma parameters for other gases used for polymer processing.
\end{abstract}

\section{INTRODUCTION}

Plasma polymerization means the fragmentation and subsequent deposition of organic precursors or monomers. It is possible to use a single gas (organic monomer gas) or a mixture of gases (organic monomer gas combined with argon or helium). This gas is fed into the reaction chamber forming the plasma that is deposited on substrate to realize film deposition.

The literature review revealed that the application of plasma-polymerized surfaces is associated with biomedical uses, as immobilized enzymes, sterilization and pasteurization, the textile industry, electronics (amorphous semiconductors), electrics (insulators, thin film dielectrics), optical applications, chemical processing (reverse osmosis membrane, permselective membrane) and surface modification (adhesive improvement, protective coating). The biomedical application of plasma polymerization mainly relies on changing the surface chemistry and tailoring the surface to be either bio reactive or nonreactive [1].

Several parameters affect plasma polymerization such as monomer flow rate, system pressure, geometry of the system, reactivity of the starting monomer, and frequency of the excitation signal.

The objective of this research paper is to develop a numerical model of an asymmetric capacitively coupled plasma $(\mathrm{CCP})$ used for polymer processing and to validate this model with experimental results for a range of operating conditions. The parameters of interest are pressure and radiofrequency voltages. The study conducted concerns the glow intensity distribution of the discharge plasma against the tube radius and report the radial profiles of electron temperature and plasma concentration in the RF capacitive discharge.

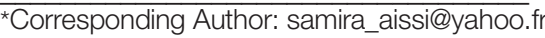




\section{DISCHARGE MODEL}

\subsection{Plasma conditions and devices}

A capacitively coupled reactor is used to carry out plasma treatments and to produce plasma polymers as shown in figure 1 .

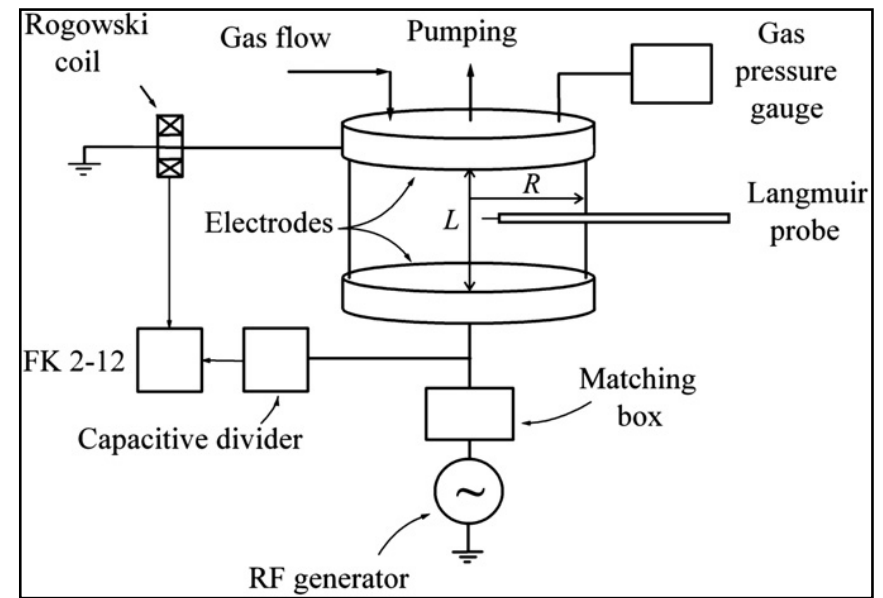

Figure 1: Scheme of the experimental device (gas: Argon, $L=0.022 \mathrm{~m}, \mathrm{R}=0.05$ m) [2]

The simulation results are performed in argon with the pressure values within the range of (0.1-2.5)Torr, the amplitude values of the RF voltage $\mathrm{Vrf}=200 \mathrm{~V}$ and the RF field frequency $\mathrm{f}=13.56 \mathrm{MHz}$. The coupling with the external circuit is realized through a blocking capacity $\mathrm{CB}=100 \mathrm{pF}$. First, a symmetric discharge chamber of $0.1 \mathrm{~m}$ in diameter with a gap between flat parallel stainless-steel electrodes of $\mathrm{L}=0.022 \mathrm{~m}$ is performed [2]. Then, an asymmetric electrodes simulation with anode diameter $(0.10 \mathrm{~m})$, cathode diameter $(0.12 \mathrm{~m})$ and gap between flat parallel stainless-steel electrodes $(0.0254)$ is realized. The RF potential is applied to one of the electrodes whereas another one is grounded. Figure 2 shows the simulation domain diagram with asymmetric geometry, already considered in the literature [3].

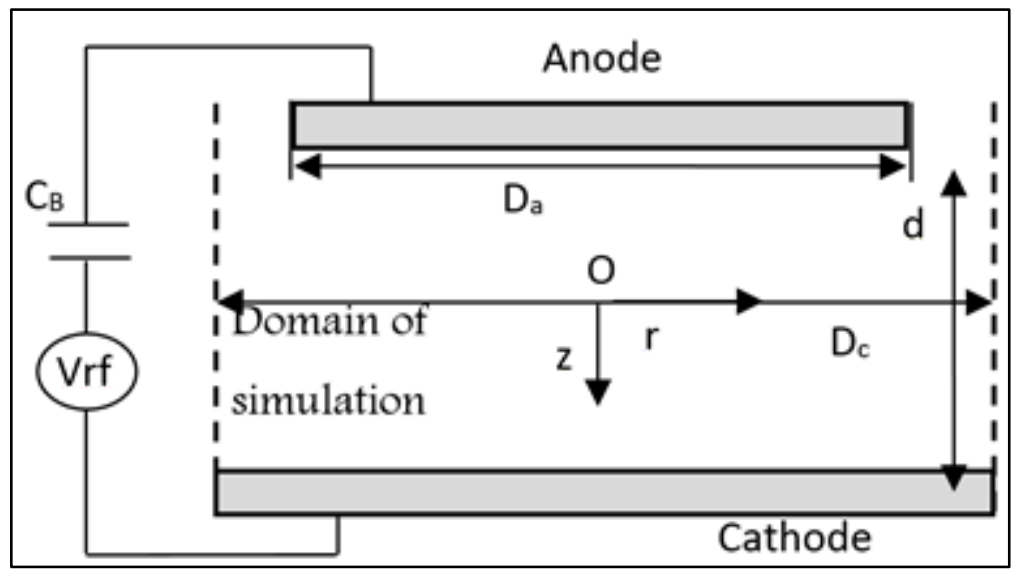

Figure 2: Schematic of the asymmetric RF discharge $(\mathrm{d}=0.0254 \mathrm{~m}, \mathrm{Da}=10 \mathrm{~cm}$ and $\mathrm{Dc}=12 \mathrm{~cm})$ [3] 


\subsection{Model formulation}

The governing equations in the fluid model are expressed below to describe the movement of charged particles formed in the plasma [4].

The continuity and momentum equations are expressed as

$$
\begin{gathered}
\frac{\partial n}{\partial t}+\nabla \cdot(n u)=S \\
\frac{\partial(m n u)}{\partial t}+\nabla \cdot(m n u u)=q n E-\nabla p-m n \vartheta_{m} u
\end{gathered}
$$

Equations (1) and (2) are used for electrons, ions and neutrals, respectively.

When pressure is not too low, equation (2) can be simplified to the drift-diffusion approximation for electrons as

$$
\Gamma=n u=-\mu n E-D \nabla n
$$

Mobility $\mu$ and diffusion coefficient D satisfy the Einstein relation. The variables n, m, q, $\mathrm{p}$, $\mathrm{u}$ and $v_{m}$ stand for the density, mass, charge, pressure, velocity and collision frequency of each particle, respectively. $\mathrm{S}$ is the source of particles generated or consumed in chemical reactions, and $\mathrm{E}$ stands for the electric field intensity vector.

The electron energy equation is expressed as:

$$
\frac{\partial}{\partial t}\left(\frac{3}{2} n_{e} k_{B} T_{e}\right)+\nabla \cdot q_{e}-e \Gamma_{e} . \nabla V=S_{\varepsilon}
$$

With the total electron energy flux $q_{e}$ given by:

$$
q_{e}=-K_{e} \nabla T_{e}+\frac{5}{2} k_{B} T_{e} \Gamma_{e}
$$

The thermal conductivity of electron $K_{e}$ is given by:

$$
K_{e}=\frac{5}{2} k_{B} D_{e} n_{e}
$$

Where ne is the electron density, $\mathrm{k}_{\mathrm{B}}$ is the Boltzmann constant and Te is the electron temperature.

Finally, electric the Poisson's Eq. (7) relates the gradient of the local electric field to the charge density, where $\varepsilon$ stands for permittivity, and $n_{+}$is the positive ion density

$$
\nabla^{2} V=-\frac{e}{\varepsilon}\left(n_{+}-n_{e}\right)
$$

The CCP discharge simulation is carried out with the COMSOL Multiphysics software [5]. The finite element method is used to numerically solve the equations. A non uniform mesh grid arrangement is chosen to cluster the points near the electrodes and the axial symmetric 
axis. Unsteady simulation is employed with time differencing of the first-order Euler scheme. The time step of electron motion is set as $1 / 20$ of RF time cycle and $4500 \mathrm{RF}$ cycles is used to attain a steady state. At each time step, Poisson's equation is solved to get the electric field distribution, and particles fluid equations are solved secondly to get the parameters such as the velocity, pressure, temperature, density for each species.

\subsection{Basic data}

The main problem concerning the correct use of the fluid model, involving the conservation equations of momentum transfer and energy under their complete form (Eqs. $(3,4))$, is due to the knowledge of the basic data.

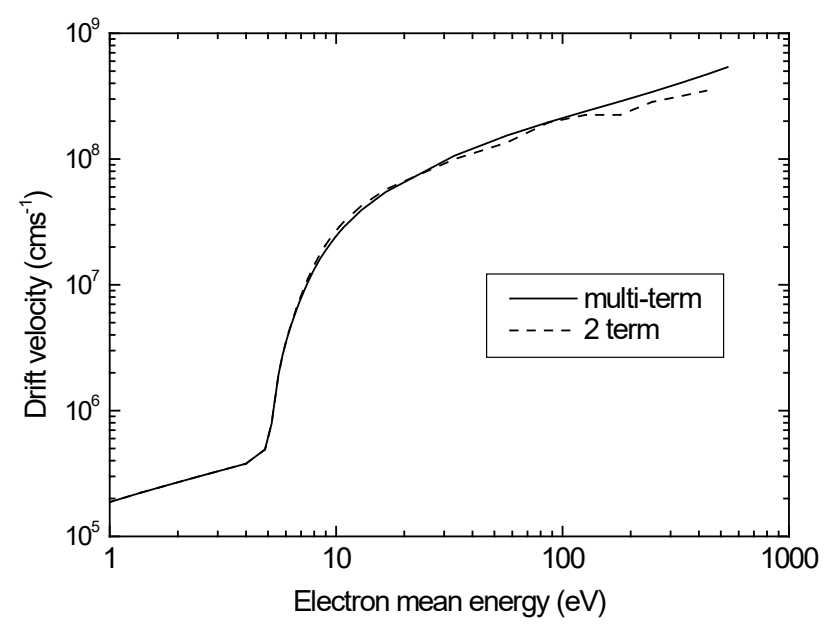

(a)

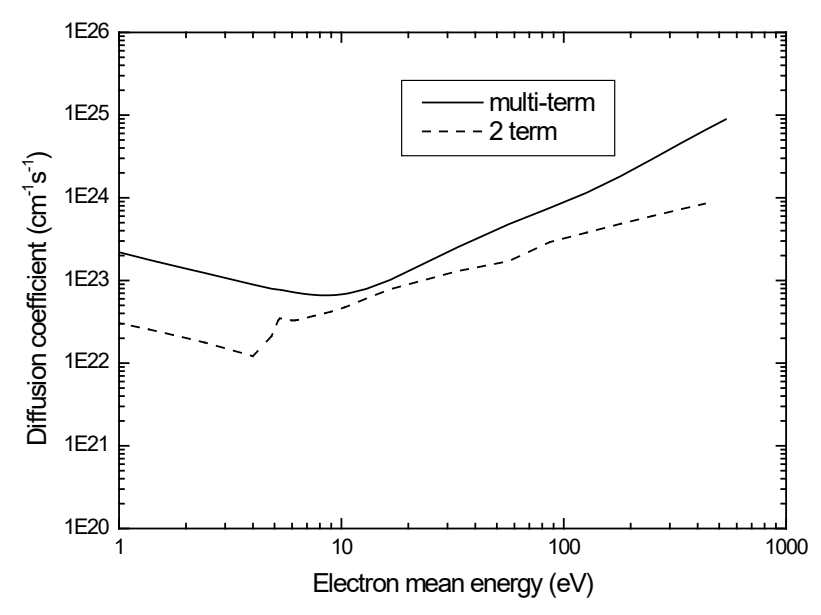

(b)

Figure 3: (a) Electron drift velocity and (b) Longitudinal electron diffusion coefficient $\mathrm{ND}$ as a function of electron mean energy in Ar gas from Boltzmann equation calculations. 
The classical basic data used for charged particles (electrons and ions) in Ar are determined from the collision cross section knowledge by using Boltzmann equation solution for electrons and Monte Carlo simulation for Ar+ ion. Figure 3 shows the electron basic data as a function of electron mean energy in Ar gas from Boltzmann equation calculations (two terms and multi-terms solutions).

In figure 4, the reduced macroscopic quantities needed for the source term of the energy conservation equation for electrons in Ar gas are presented as a function of electron mean energy from Boltzmann equation calculations.

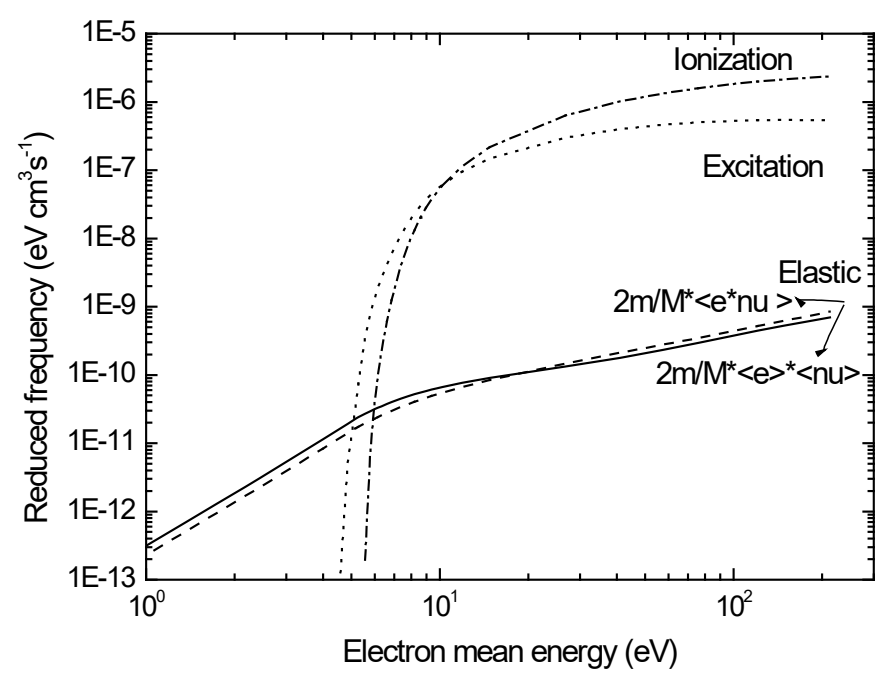

Figure 4: Reduced macroscopic quantities for electrons in Ar gas as a function of electron mean energy from Boltzmann equation calculations.

\section{RESULTS AND DISCUSSION}

Using the previous basic data, the second order model is successfully validated in one dimensional model in the case of a RF glow discharge in Ar treated by Lin and Adomaitis [6] under their operating discharge parameters.

\subsection{Symmetric Configuration}

In the case of an RF discharge established between two parallel planar electrodes, a twodimensional model is adopted to simulate the electrical and energetic characteristics of the RF reactor. The densities of the charged particles as well as the solution of the Poisson equation are obtained for an inter-electrode gap of $0.022 \mathrm{~m}$, a pressure of $100 \mathrm{mT}$ Torr, a voltage of $200 \mathrm{~V}$ and a frequency of $13.56 \mathrm{MHz}$.

Figure 5 shows that, electrons are able to follow instantaneously variations of the electric field because of their low inertia. During the evolution of the radiofrequency cycle, electrons are attracted to the left electrode (momentarily anode) at the beginning of the RF cycle giving a negative space charge which decreases the electric field in the vicinity of this electrode. The electrons are pushed back to the plasma region which leads to a positive space charge in the sheath. This appearance of the positive charge at vicinity of the electrode, momentarily 
cathode, leads to the increase of the electric field at this location causing the expansion of this sheath. The movement of electrons by covering and discovering the positive space charge in the sheaths is the basis formation and oscillation of the sheaths.

However, ions are not sensitive to the temporal evolution of the electric field during the cycle. Ion transport is rather affected by a middle electric field called an effective field. For a given RF cycle, the variation of the density of the positive ions is only a function of the position.

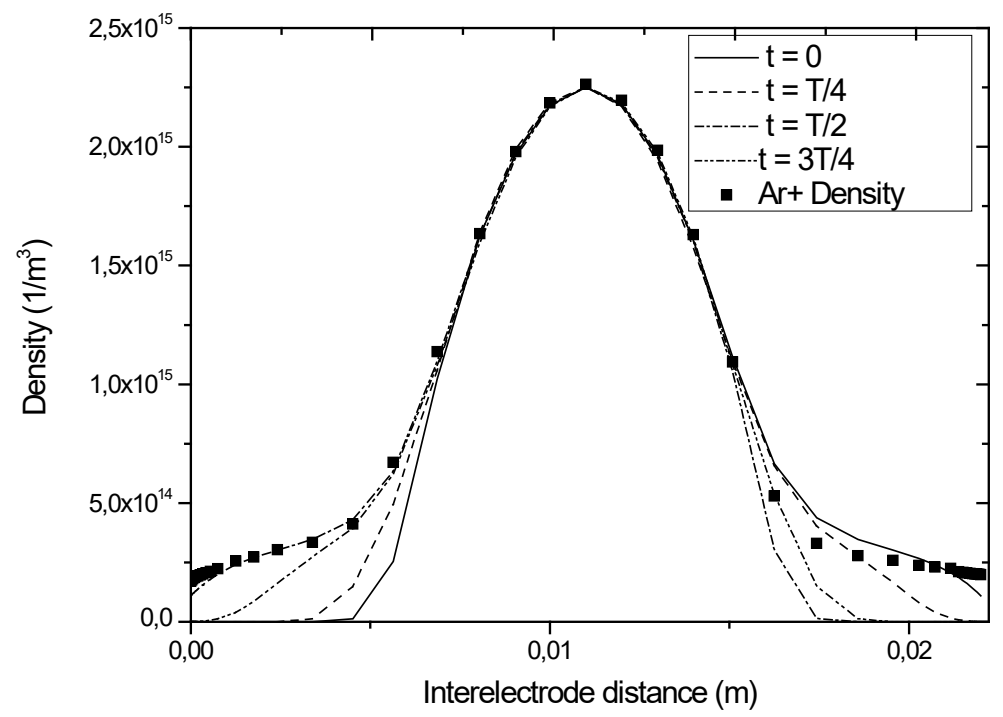

Figure 5: Density distributions along a $z$ axis for the 2D symmetric reactor $(p=100 m$ Torr, $V r f=200 V)$

Figure 6 shows the spatiotemporal variation potential in the discharge during the RF cycle. The tension radiofrequency $\operatorname{Vrf}(t)$ is applied to the upper electrode $(z=0.022 \mathrm{~m})$ while the electrode down is grounded $(\mathrm{z}=0 \mathrm{~m})$. The potential in the plasma region is an effective barrier to maintain plasma stability and neutrality. Its Value is always greater than that of the electrodes region. In fact, this average value is close to half of the peak voltage applied because the self-bias voltage is zero in the case of a symmetrical discharge.

Figure 7 represents the spatiotemporal evolution of the temperature of electrons over an $\mathrm{RF}$ cycle. In the sheath plasma, the temperature of the electrons is controlled by the expansion and contraction of the sheaths. In other words, average energy electrons that is directly related to the electric field amplitude, increases when the contraction of the sheath and decreases during the expansion of the sheath. The average energy is low and almost constant in the plasma region. 


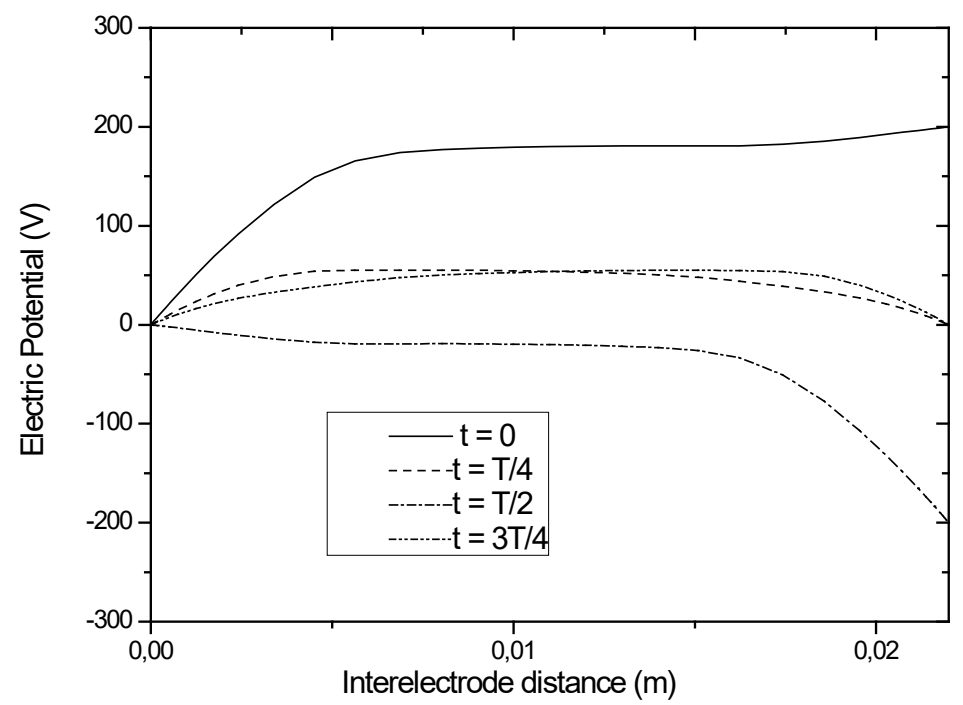

Figure 6: Potential distributions along a $z$ axis for the 2D symmetric reactor $(\mathrm{p}=100 \mathrm{mTorr}, \mathrm{Vrf}=2 \mathrm{OOV})$

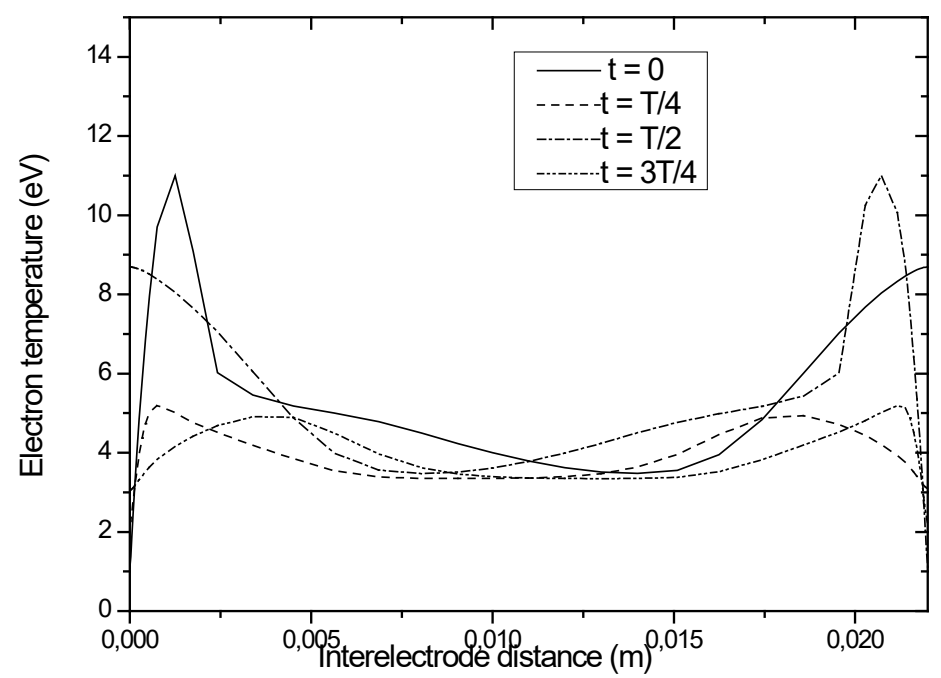

Figure 7: Electron temperature distributions along a $z$ axis for the 2D symmetric reactor $(p=100 m$ Torr, $\mathrm{V} r f=200 \mathrm{~V})$ 


\subsection{Radial structure of low pressure RF capacitive discharges}

The radial structure of the RF discharge is of considerable interest to possess a uniform distribution of plasma parameters across the electrode (plate) area.

Figure 8 shows the radial profiles of the electron temperature for two values of the argon pressure (figure $8(\mathrm{a}): \mathrm{p}=0.1$ Torr and figure $8(\mathrm{~b}): \mathrm{p}=1$ Torr) and various $\mathrm{RF}$ voltage values registered in the central plane of the discharge. It is clear that in the weak-current $\alpha$-mode of the RF discharge (the curve for $\mathrm{Vrf}=100 \mathrm{~V}, \mathrm{p}=0.1$ Torr), the electron temperature remains constant and increases on approaching to the wall of the discharge tube.

On increasing the RF voltage, the electron temperature in the $\alpha$-mode [7] decreases weakly in the total discharge volume but the peak of the electron temperature near the tube wall still remaining. The changing radial electric field, heat electrons and increase plasma concentration near the boundary of the radial sheath.

Under the $\alpha-\gamma$ transition of the RF discharge, the magnitude of the electron temperature peak near the tube wall decreases abruptly (the curve for $\operatorname{Vrf}=200 \mathrm{~V}, \mathrm{p}=1$ Torr). Then, in the strong-current $\gamma$-mode, this peak of electron temperature is absent.

A good agreement is observed between calculated results and the experimental results registered with a Langmuir probe from the literature [2] for the two-discharge mode.

\subsection{Asymmetric model}

In order to analyse the asymmetric effect, the results are obtained for an argon discharge in a cylindrical reactor, with $\mathrm{Da}=10 \mathrm{~cm}, \mathrm{Dc}=12 \mathrm{~cm}$ and $\mathrm{d}=2.25 \mathrm{~cm}$ under tension radio frequency of $200 \mathrm{~V}$ peak ( $400 \mathrm{~V}$ peak-to-peak), $13.56 \mathrm{MHz}$ frequency and on argon gas pressure of $100 \mathrm{mT}$ Torr.

The electron and ions density is shown in figure 9. These densities are almost equal in the plasma region (which shows the neutrality of this medium) with a slightly larger value next to the side grid. This is due to the double expansion and contraction of the radial and axial sheaths, caused by the temporal variation of the voltage of the radiofrequency source. Contrary to symmetric case, the difference in amplitude between electron density and ion density appear in the sheaths. Because of the self-bias potential, the symmetry of the sheaths in geometrically asymmetric discharges can be broken.

Figure 10 shows calculated 2D time averaged ion density of Ar+.

From figure 11, the maximum radial density is located in the plasma region at the edge of the discharge in the part asymmetrical $(\mathrm{Dc}=12 \mathrm{~cm})$. In the centre of the discharge, densities are approximately 2 times lower than found in symmetric systems.

Due to the geometrical asymmetry, the time averaged sheath voltage and width are larger at the smaller powered electrode compared to the larger grounded electrode. Therefore, the electron energy is strongly affected (figures 12-13).

The results obtained are compared with the experimental results from the literature $[8,9]$. The figure 14 shows the electron density profiles calculated at a pressure of $100 \mathrm{mTorr}$, for different voltages radio frequencies. There is a good agreement between our calculations and measurements. These agreements are the sign of the coherence and validity of the developed model in asymmetric geometry. 


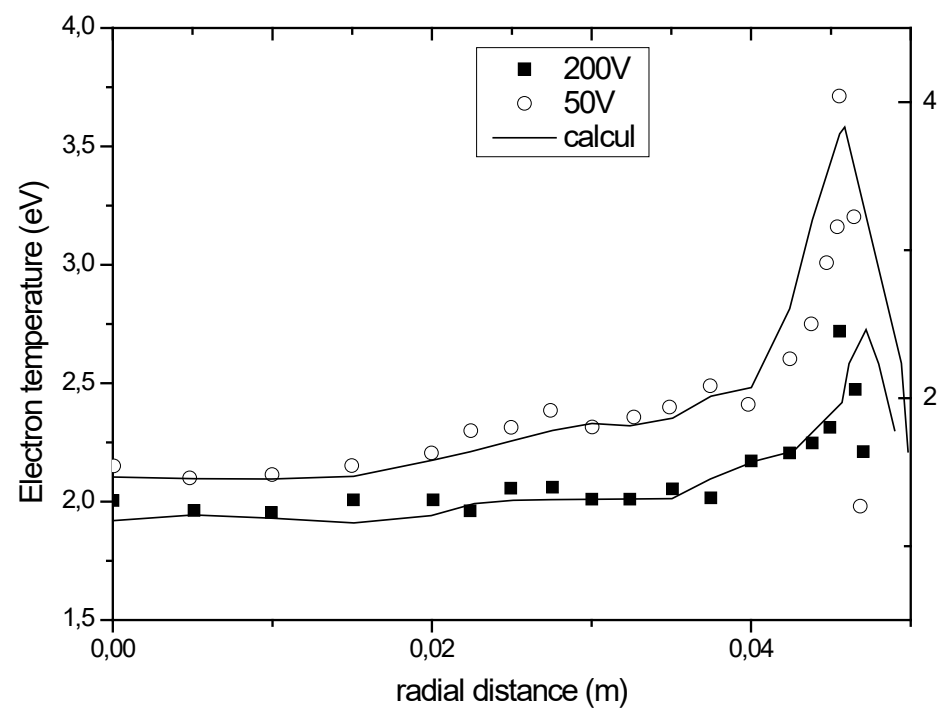

(a)

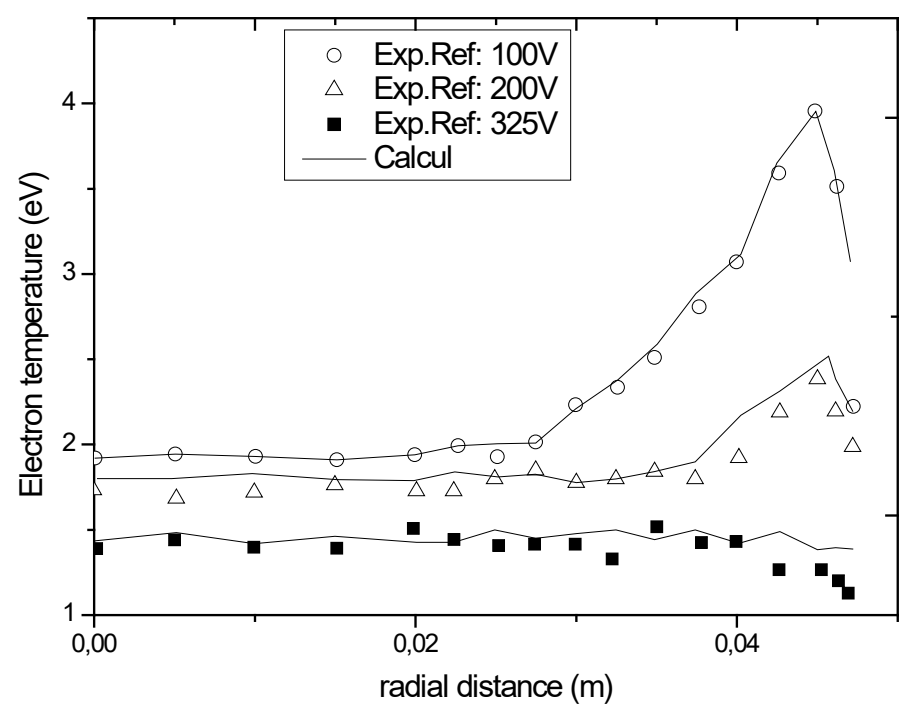

(b)

Figure 8: Radial profiles of electron temperature with argon pressure of 0.1 Torr (a) and 1 Torr (b) and various RF voltage values. The inter-electrode gap is $0.022 \mathrm{~m}$, the chamber diameter is $0.1 \mathrm{~m}$. 


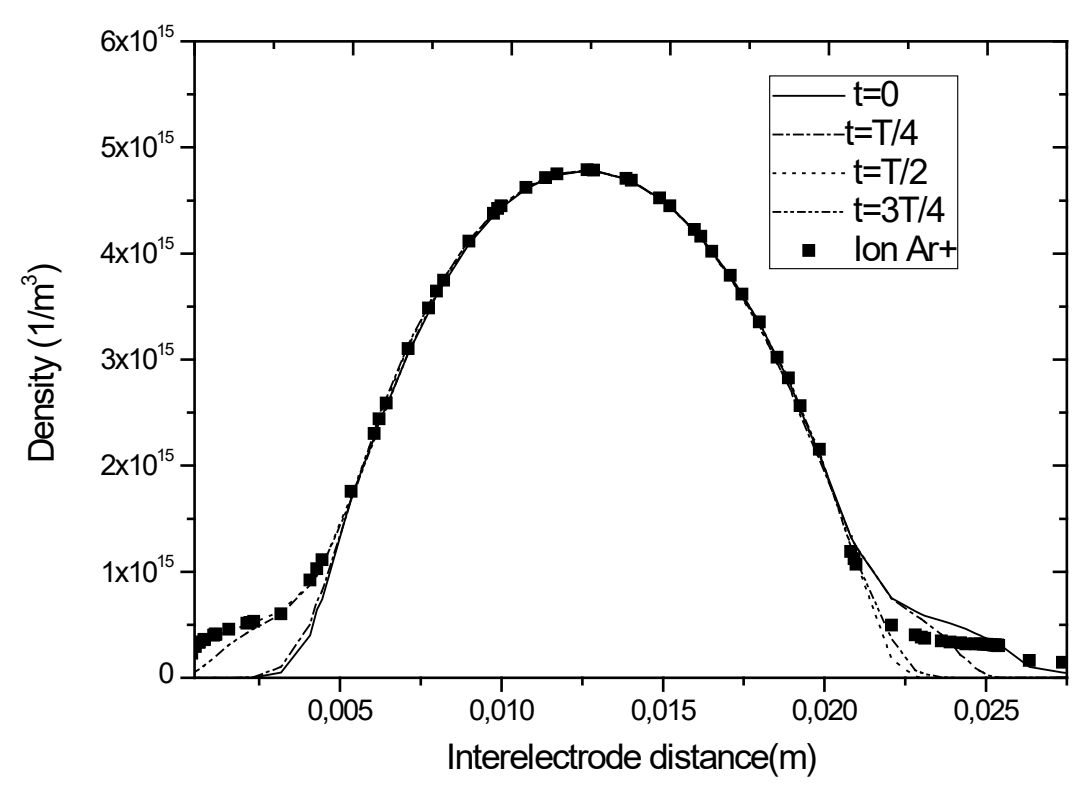

Figure 9: Density distributions along a $z$ axis for the 2D asymmetric reactor $(p=100 m$ Torr,, $\mathrm{rf}=200 \mathrm{~V})$

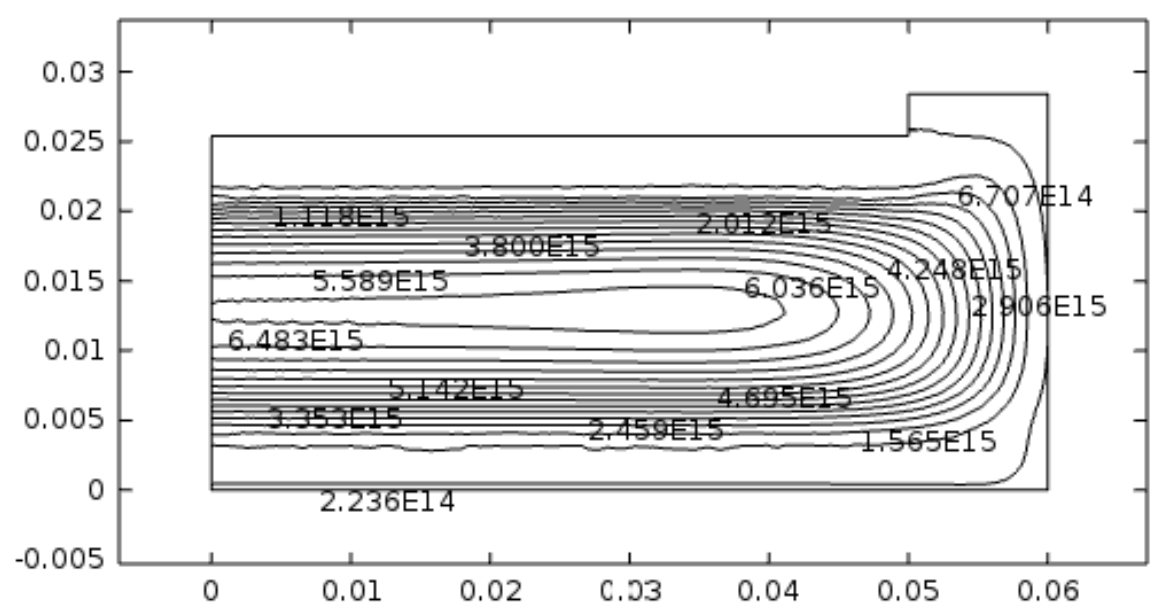

Figure 10: Calculated 2D time averaged ion density of Ar+ 


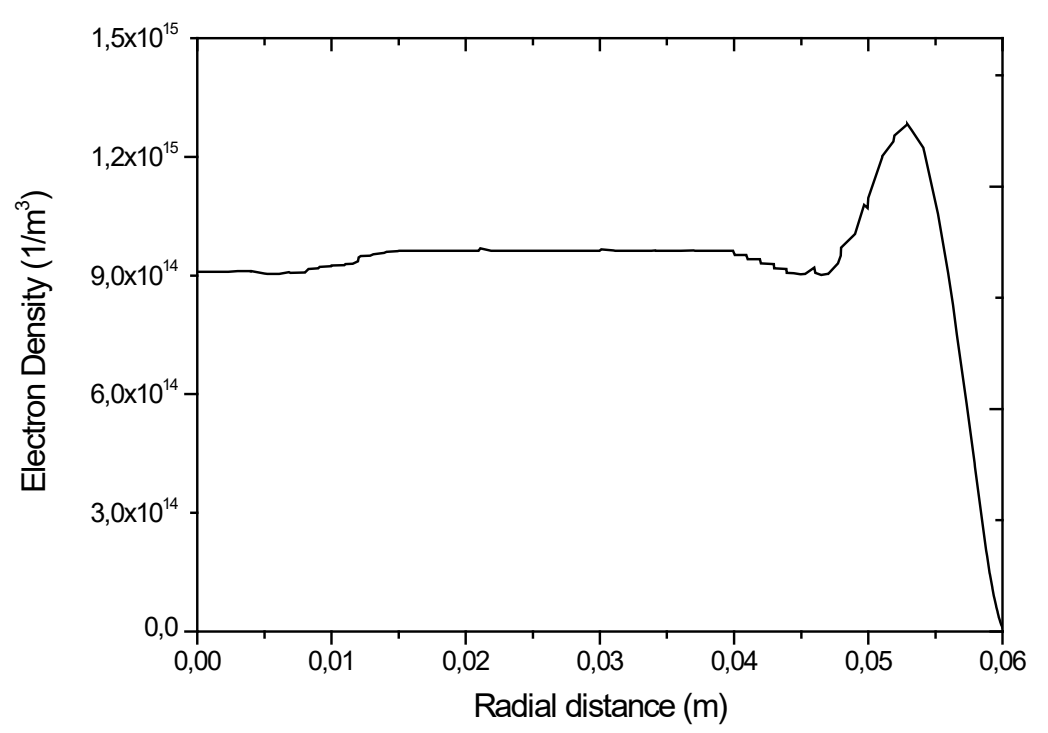

Figure 11: Radial Density distributions for the 2D asymmetric reactor $(\mathrm{p}=100 \mathrm{mTorr}, \mathrm{Vrf}=200 \mathrm{~V})$

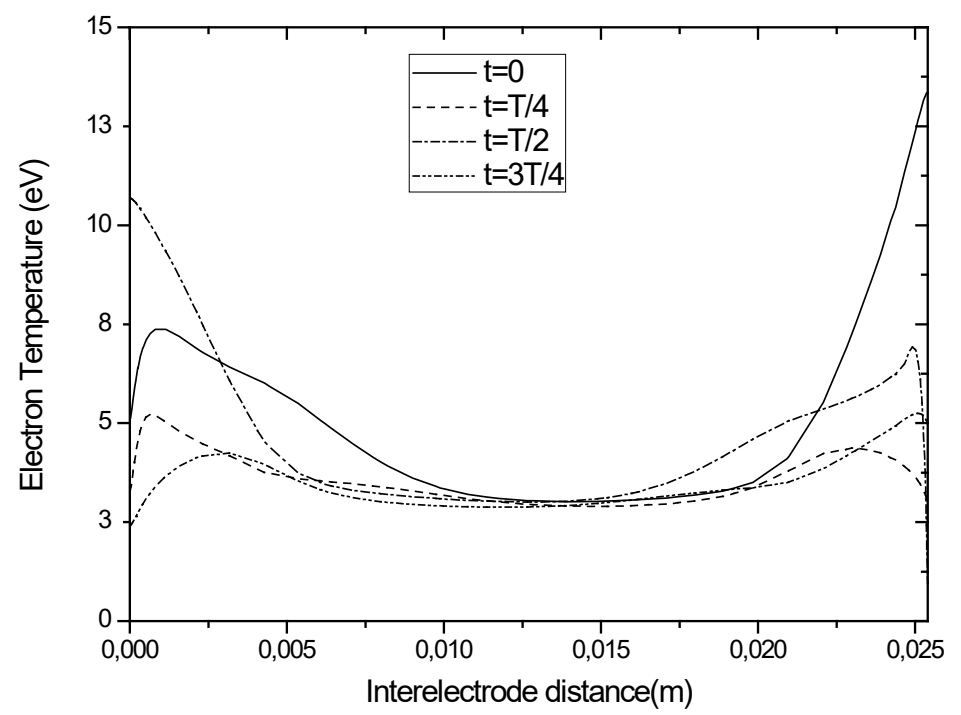

Figure 12: Electron temperature distributions along a $z$ axis for the $2 \mathrm{D}$ asymmetric reactor $(p=100 \mathrm{mTorr}, \mathrm{V} r \mathrm{f}=200 \mathrm{~V})$ 


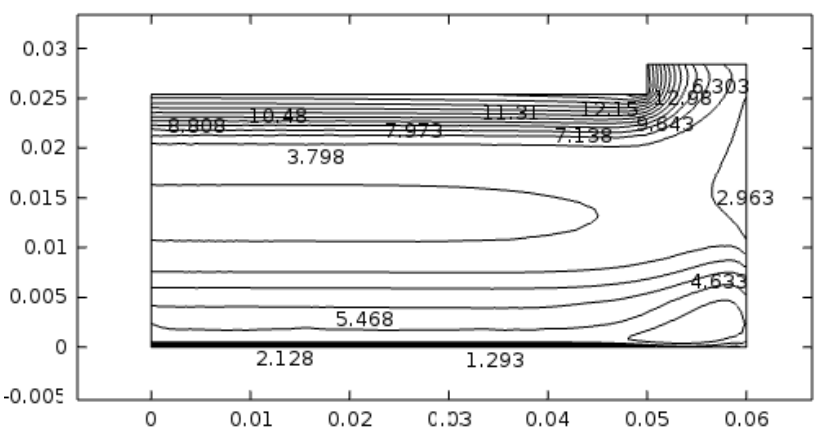

(a)

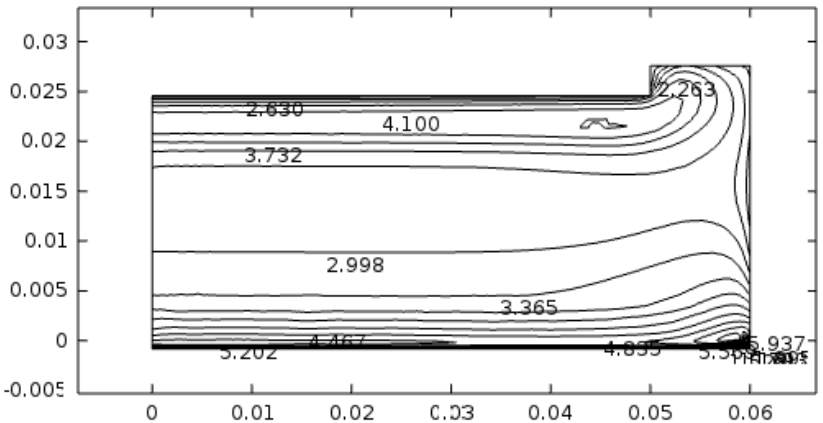

(b)

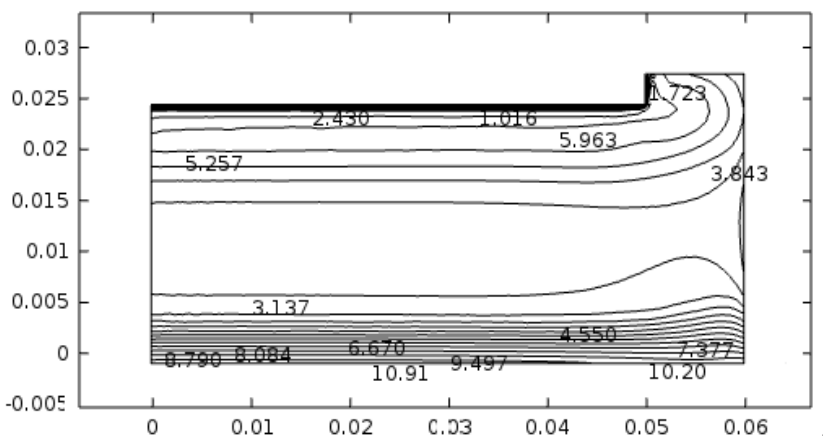

(c)

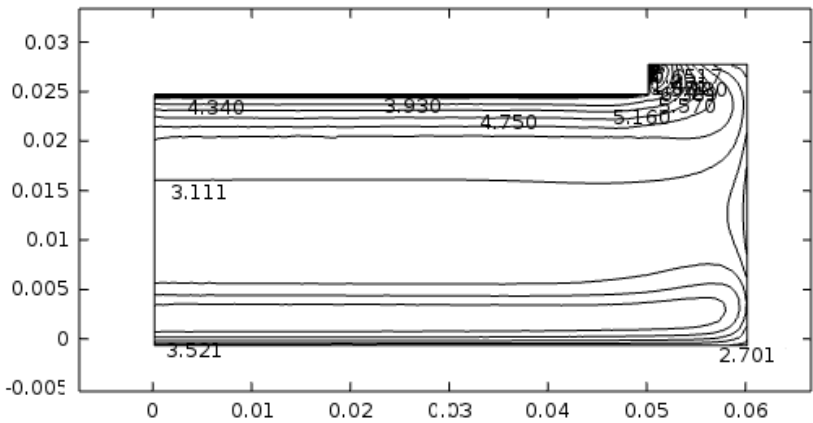

(d)

Figure 13: Calculated 2D electron temperature profiles at the four different phases in the rf cycle ((a)t=0, (b) $t=T / 4,(c) t=T / 2$, (b)t=3T/4) 


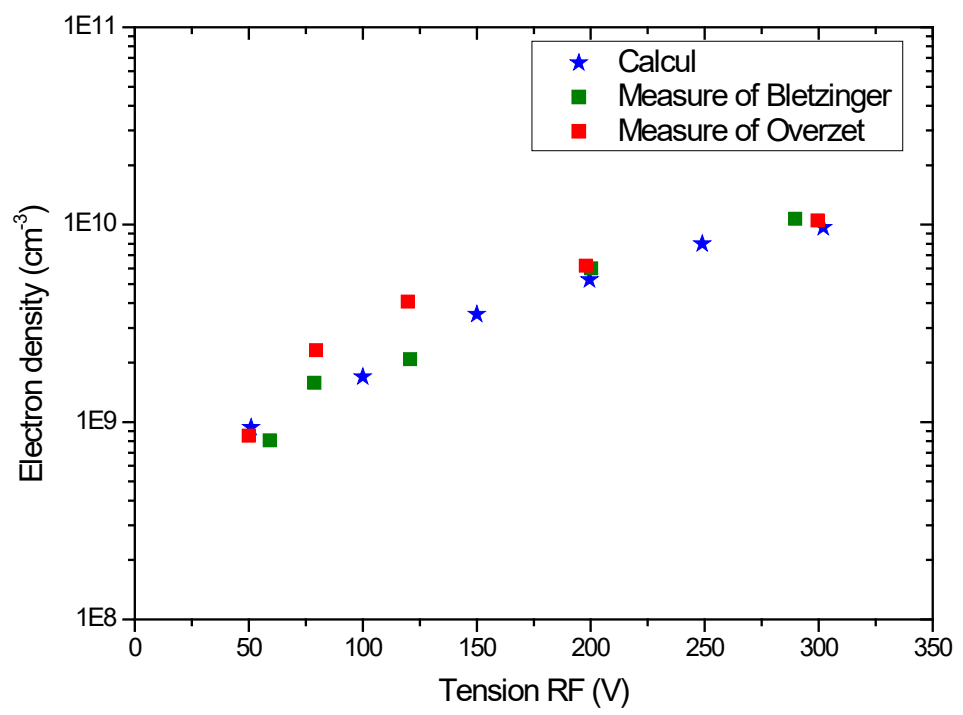

Figure 14: Comparison between the calculated results and the experimental results of electron density by varying the radiofrequency voltage

\subsection{Depositing Monomers}

The plasma polymerization involves plasmas of complex organic vapors, which have a larger range of species including radicals, ionic oligomers and fragmented neutrals $[10,11]$.

For this purpose, two different gases (argon and oxygen) are used and compared for an electrode separation of $22 \mathrm{~cm}$ at a constant gas pressure of $100 \mathrm{mTorr}$.

The behavior of self-bias potential as function of the RF power taken from the generator, plotted in figure 15, shows only slight variation between two gases, particularly in the low power region, where the most functionalized coatings are produced.

Figure 16 shows calculated ions flux as a function of RF power plasma for argon and oxygen.

The agreement shown with experimental results [12] for depositing monomers and nonpolymerizing gases is highly encouraging. Finally, the model results for argon obtained can be used for other gases. 


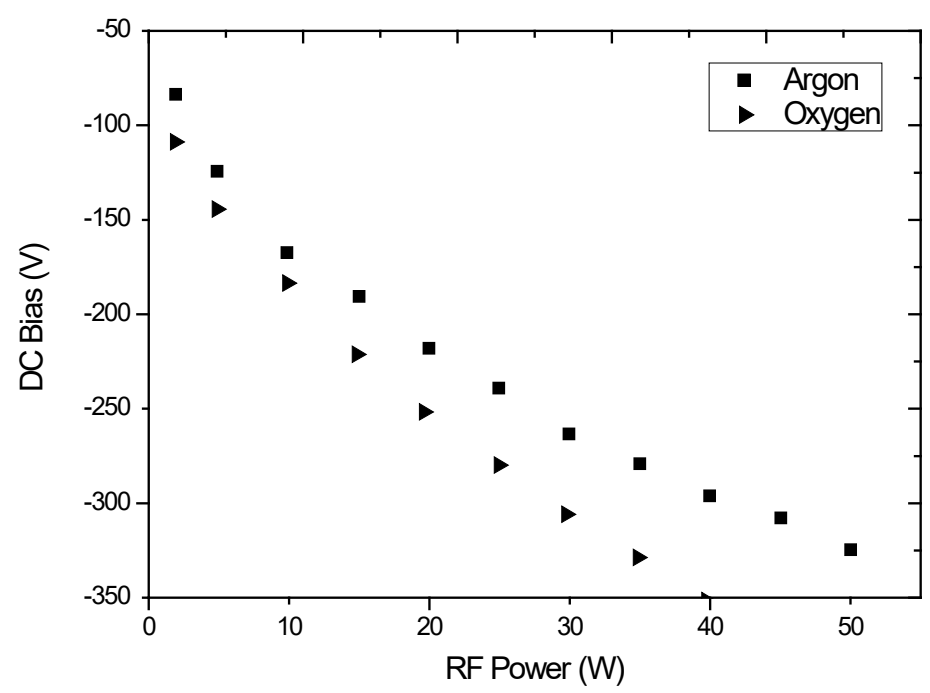

Figure 15: Variation of cathode dc bias with RF power plasma

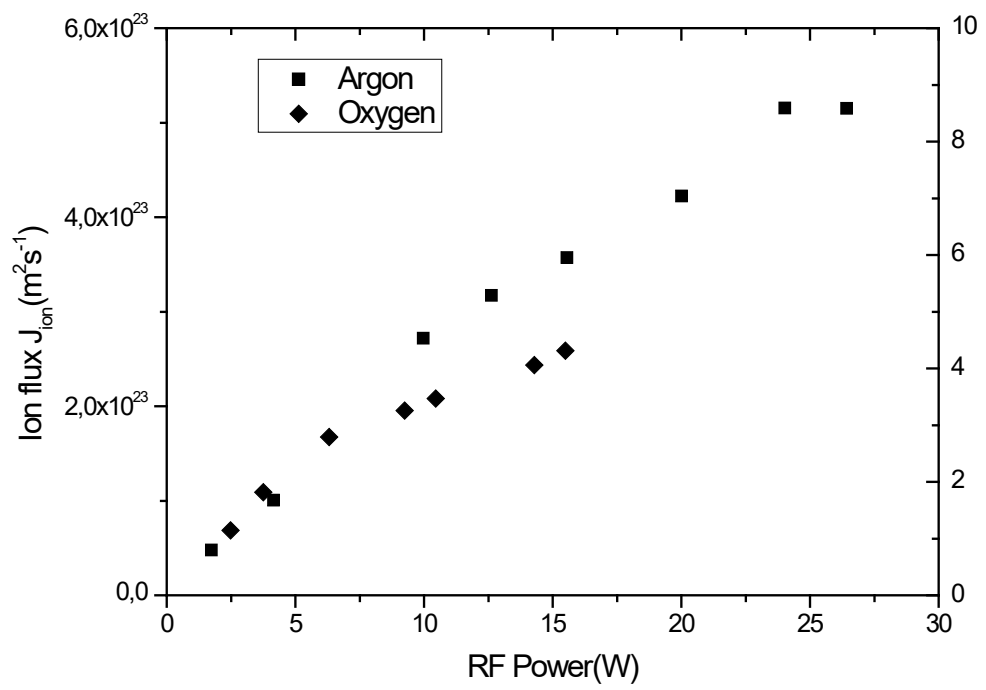

Figure 16: Calculated ions flux as a function of RF power plasma 


\section{CONCLUSION}

A numerical global model is developed for argon to describe capacitively coupled radiofrequency (CCRF) discharges used to produce plasma polymers. The model describes various discharge types, as well as geometrically symmetric and asymmetric discharges [13, 14].

The radial distribution of the electron temperature near the tube wall in the RF capacitive discharge is revealed. In the $\gamma$-mode of the rf capacitive discharge, the electron temperature decreases in the total plasma volume, and the peak of the glow intensity near the tube wall disappears.

In asymmetric geometry, the self-bias voltage and, consequently, the sheath widths and potential profile are effectively affected.

Finally, modeling the reactor for argon and oxygen plasma shows only slight variation between two gases, particularly in the low power region, where most functionalized coatings are produced. Thus, the modelling of the reactor with Ar plasma can predict the plasma parameters for deposition of organic precursors or monomers used in plasma polymerization processes when the RF power transfer efficiency is taken into account.

\section{REFERENCES}

[1] Safizadeh H., et al., Particle aggregates formed during furfuryl methacrylate plasma polymerization affect human mesenchymal stem cell behavior, Colloids and Surfaces B: Biointerfaces, 2018. 161(261): p. 261-268.

[2] Lisovskiy, V.A., N.D. Kharchenko, and V.D. Yegorenkov, Radial structure of low pressure rf capacitive discharges, 2010.Vacuum 84: p. 782-791.

[3] Benyoucef, D., M. Yousfi, B. Belmadani and A. Settaouti, PIC MC Using Free Path for the Simulation of Low-Pressure RF Discharge in Argon, IEEE Transactions on plasma science, 2010. 38(4): p. 902-908.

[4] Elaissi, S., and al., Electric discharge modelling with the contribution of convection and drift energy effects, Eur.Phys. J.Apply.Phys, 2005. 32: p. 37-44.

[5] COMSOL Multiphysics ${ }^{\circledR}$ Software, https://www.comsol fr, 2018.

[6] Lin, Y.H., and R. Adomaitis, A global basis function approach to DC glow discharge simulation, Technical Research Report, Institute for Systems Research ,1997. T.R. 978: P.1-13

[7] Shi, J.J., and M.G. Konga, Mechanisms of the $\alpha$ and $\gamma$ modes in radio-frequency atmospheric glow discharges, Journal of Applied Physics, 2005. 97, p. 023306-1023306-6.

[8] Bletzinger, P., and A. Garscadden, IEEE Conference on Plasma Science, Williamsburg, VA, June 3-5, 1991.

[9] Overzet, L.J., Microwave Diagnostic Results from the Gaseous Electronics Conference RF Reference Cell, Journal of Research of the National Institute of Standards and Technology, 1995. 100: p. 401-414.

[10] Schüngel, E., and al., Control of plasma properties in capacitively coupled oxygen discharges via the electrical asymmetry effect, Journal of Physics D: Applied Physics, 2011. 44(28): p. 1-22. 
[11] Liu, Y.X., and al., Striations in electronegative capacitively coupled radio-frequency plasmas: Effects of the pressure, voltage, and electrode gap, Physics of Plasmas, 2017. 24, 073512: p. 1-32.

[12] Michelmore, A., and al., An Experimental and Analytical Study of an Asymmetric Capacitively Coupled Plasma used for Plasma Polymerization, Plasma Process and Polymers, 2014. 11(9): p. 833-841.

[13] Shulze, J., E. Schüngel and U. Czarnetzki, The electrical asymmetry effect in capacitively coupled radio frequency discharges - measurements of DC self bias, ion energy and ion flux, Journal of Physics D: Applied Physics, 2009. 42 (9).

[14] Bruneau, B., and al., Slope and amplitude asymmetry effects on low frequency capacitively coupled carbon tetrafluoride plasmas, Journal of Applied Physics, 2016. 119, 163301: p. 163301-1 - 163301-10. 